\title{
Diffuse Corneal Edema after Uneventful Pterygium Surgery: Toxic Anterior Segment Syndrome or Toxic Keratopathy?
}

\author{
(1) Ceyhun Arıcı*, (1) Burak Mergen**, (1) Oğuzhan Kılıçarslan*, (1) Ahmet Ağaçhan***, \\ (1) Beril Tülü Aygün***, (1) Akif Özdamar* \\ *İstanbul University-Cerrahpaşa, Cerrahpaşa Faculty of Medicine, Department of Ophthalmology, İstanbul, Turkey \\ **University of Health Sciences Turkey, Başakşehir Çam and Sakura City Hospital, Clinic of Ophthalmology, İstanbul, Turkey \\ ***University of Health Sciences Turkey, İstanbul Beyoğlu Eye Training and Research Hospital, İstanbul, Turkey
}

\begin{abstract}
A 29-year-old woman was referred to our department for corneal edema after uneventful pterygium excision surgery with conjunctival autografting. She was prescribed topical dexamethasone and showed a complete response within 2 weeks of treatment. Specular microscopic examination revealed severe endothelial cell loss in the operated eye. Mild corneal haze causing a decrease in vision (20/50) was observed in long-term follow-up. This steroid-responsive complication was linked to two possible etiologies: mild toxic anterior segment syndrome or povidone-iodine (PVP-I) corneal toxicity. Surgeons should be careful during pterygium surgery to completely clear PVP-I and avoid any penetration into the anterior chamber to prevent possible serious complications. When diffuse corneal edema is encountered after pterygium surgery, intense steroid treatment should be prescribed as in the present case.
\end{abstract}

Keywords: Pterygium, povidone iodine, toxic anterior segment syndrome, toxic keratopathy

\section{Introduction}

Pterygium is a wing-shaped fibrovascular proliferation of conjunctiva over the cornea, mostly on the nasal side. Its prevalence was reported to be between $3 \%$ and $30 \%$ in different countries. ${ }^{1}$ The bare sclera technique or using a conjunctival or conjunctivolimbal autograft or amniotic membrane graft with suturing or fibrin glue are among the possible surgical strategies for pterygium and have different recurrence rates. ${ }^{2}$ To prevent recurrence, antimitotic agents such as mitomycin C (MMC) can be applied during surgery or as postoperative eye drops. ${ }^{2}$ However, MMC may have toxic effects on the cornea, including corneal epithelial toxicity or edema and scleral melting, with increased risk at higher dosage. ${ }^{3}$

Although rare, some minor complications such as wound dehiscence, Tenon's granuloma, and conjunctival cyst might develop after surgical excision of pterygium. However, diffuse corneal edema similar to toxic anterior segment syndrome (TASS) has not been reported in the literature.

Here, we report a case of diffuse corneal edema resembling toxic keratopathy or TASS after uneventful pterygium surgery involving a sutured conjunctivolimbal autograft with no antimitotic agent (e.g., MMC) and discuss the possible etiologies.

Address for Correspondence: Burak Mergen, University of Health Sciences Turkey, Başakşehir Çam and Sakura City Hospital, Clinic of Ophthalmology, İstanbul, Turkey E-mail: burakmergen@gmail.com ORCID-ID: orcid.org/0000-0002-8132-495X

Received: 19.01.2021 Accepted: 28.09.2021

Cite this article as: Arıcı C, Mergen B, Kılıçarslan O, Ağaçhan A, Tülü Aygün B, Özdamar A. Diffuse Corneal Edema after Uneventful Pterygium Surgery: Toxic Anterior Segment Syndrome or Toxic Keratopathy?. Turk J Ophthalmol 2022;52:69-71

${ }^{\circledR}$ Copyright 2022 by Turkish Ophthalmological Association 


\section{Case Report}

A 29-year-old woman presented with corneal edema after an uneventful pterygium excision surgery with conjunctival autografting. Best corrected visual acuity (BCVA) was counting fingers at 3 meters in her right eye (RE) and 20/20 in her left eye (LE). In the slit-lamp examination, diffuse corneal edema and Descemet folds were observed together with an intact graft which was upside down; conjunctiva was hyperemic in the RE (Figure 1a). Her fundus could not be examined because of corneal edema. She had been prescribed topical moxifloxacin, dexamethasone, eye lubricant, and $10 \% \mathrm{NaCl}$ solution 3 times a day for 1 week before referral to our department.

The surgeon who performed her operation was contacted to determine the technique and ocular disinfection method he used. He stated that he applied 5\% povidone-iodine (PVP-I) (diluted in balanced salt solution) for 3 minutes and then washed the eye for preoperative antisepsis. He did not report the use of any antifibrotic agent such as MMC during the surgery. The autograft taken from the superior area had been secured to the excised nasal area with 8.0 vicryl sutures. He reported corneal edema on the first postoperative day. She had been operated by the same surgeon at the same hospital using the same technique on her LE 1 year earlier with no complications.

Specular microscopic imaging showed that the healthy eye had 2882 cells $/ \mathrm{mm}^{2}$, the RE was not clear enough to obtain a good result as depicted in the anterior segment optical coherence tomography (OCT) image (Figure 2a). The patient was prescribed topical dexamethasone (Maxidex ${ }^{\circledR}$, Alcon, USA) every hour, moxifloxacin (Vigamox ${ }^{\circledR}$, Alcon, USA) 3 times a day, and eye lubricant (Refresh ${ }^{\circledR}$, Allergan, USA) 4 times a day. After observation of a 3-mm epithelial defect on the nasal cornea after the first day of treatment, the topical dexamethasone regimen was changed to 4 times a day, a bandage contact lens (Purevision HD, Bosch \& Lomb, USA) was applied, and oral vitamin C (1 g/day) was added. After one week of treatment, a dramatic decrease in the corneal edema was observed. Descemet folds, conjunctival hyperemia, and epithelial defect disappeared. The bandage contact lens was removed and topical moxifloxacin was stopped. The corneal edema disappeared totally after 2 weeks of this treatment (Figure $1 \mathrm{~b}$ ) and the BCVA increased to 20/50. After 1 year of follow up, specular microscopy showed the endothelial cell density in the RE was 1001 cells $/ \mathrm{mm}^{2}$ (coefficient of variation [CV]: 36; hexagonality [HEX]: 46\%) and that of the LE was 2880 cells/mm² (CV: 38, HEX: 67\%). BCVA in her RE was still 20/50. Corneal stromal vascularization and corneal haze on the nasal side were observed at the last visit (Figure 1c,d) with no corneal edema (Figure 2b).

\section{Discussion}

Although minor complications can sometimes be observed after pterygium surgery, in this case we observed diffuse corneal edema. We suspected TASS or endothelial toxicity (toxic keratopathy) due to the use of PVP-I, MMC, or any solution used perioperatively as possible etiologies. However, MMC was not used during or after the surgery.

TASS is postoperative anterior segment inflammation with diffuse corneal edema that can occur after any interventions involving contact with the anterior chamber. Substances that can cause TASS include residual disinfection or sterilization solutions, preservatives in medicines, or bacterial endotoxins. ${ }^{4}$ Intense topical steroid therapy is indicated for this syndrome. In terms of TASS, although we did not observe any anterior segment reaction, we might have examined the patient at a stage when the reaction was suppressed but the corneal edema persisted. The patient's complete response to 2 weeks of steroid treatment supports this possibility.

PVP-I at a concentration of $0.025 \%$ did not show any toxic effect on human corneal endothelium in vivo when used to treat

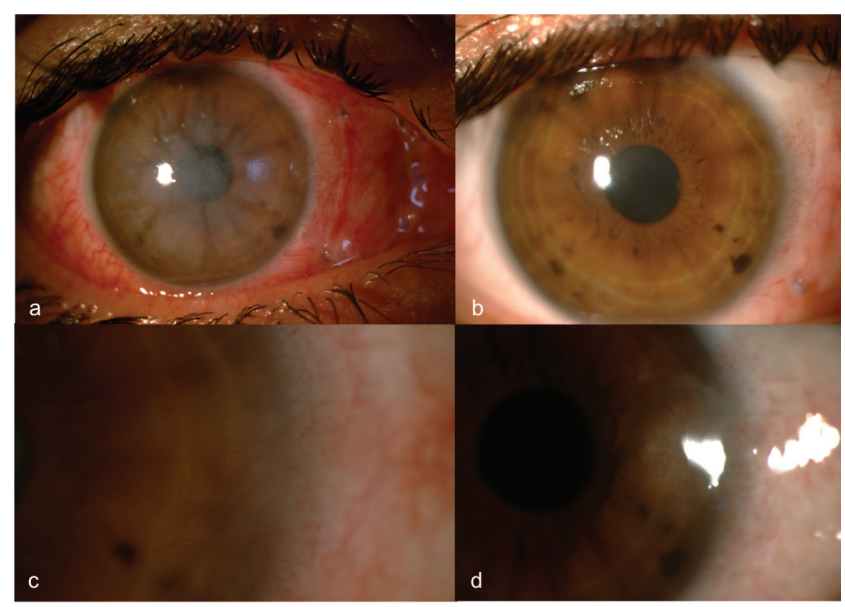

Figure 1. Biomicroscopic images of the patient showing conjunctival hyperemia and corneal edema together with the autograft at initial presentation (a), resolution of the corneal edema after 2 weeks of treatment (b), and development of corneal stromal vascularization (c) and corneal haze (d) on the nasal side after 1 year of treatment with low-dose topical steroid

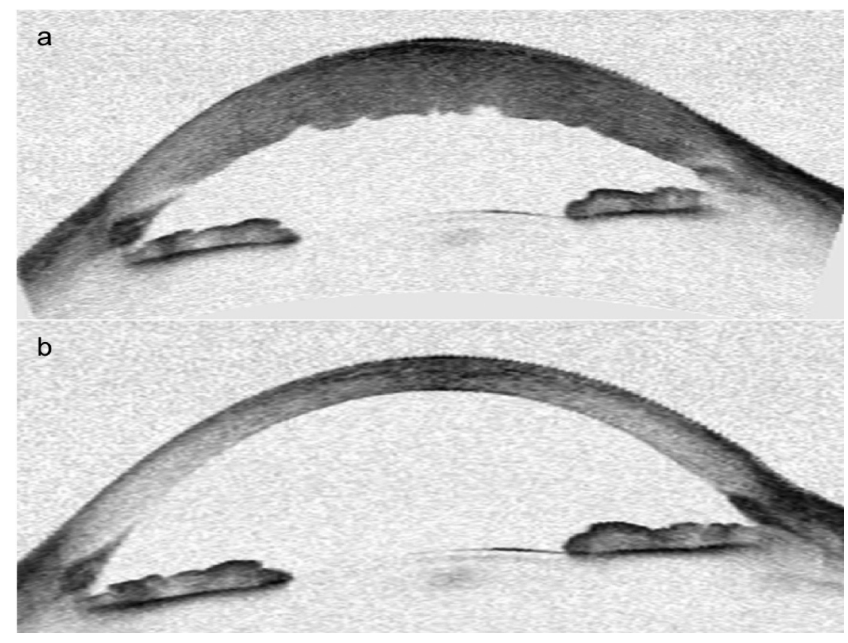

Figure 2. Anterior segment optical coherence tomography images of the patient showing diffuse corneal edema and Descemet folds at initial presentation (a) and regression of the corneal edema after 2 weeks of steroid therapy (b) 
postoperative endophthalmitis. ${ }^{5}$ However, a single drop of $5 \%$ PVP-I was shown to exert toxic effects on the corneal endothelium of healthy rabbits when injected into the anterior chamber. ${ }^{6}$ It was also shown to cause endothelial toxicity when injected at a concentration of $1 \%$ into bovine corneal endothelium. ${ }^{7}$ In their study on rabbit eyes, Jiang et al. ${ }^{8}$ postulated that although $5 \%$ PVP-I does not cause endothelial toxicity through an intact cornea when instilled on the ocular surface, it may penetrate when the epithelial layer is damaged. Therefore, 5\% PVP-I in our patient might have penetrated through the excised pterygium area to cause endothelial toxicity, since that area was devoid of corneal epithelium. The lower endothelial cell count in that eye supports this possibility. Epithelial debridement has been linked to an increased penetration of some molecules such as riboflavin. ${ }^{9}$ However, further studies are necessary to investigate the penetration of PVP-I after corneal epithelial debridement. In addition, there is no report on humans or animals showing that corneal edema secondary to the endothelial toxicity of PVP-I responds to topical steroid therapy. Although our patient showed a complete response after 2 weeks of topical steroid, corneal haze and stromal vascularization developed and persisted for 1 year despite low-dose steroid treatment.

Residual liquid disinfectants such as alcohol, glutaraldehyde, or chlorhexidine on surgical instruments might be responsible for toxic keratopathy or TASS. ${ }^{10}$ However, the surgeon who performed the procedure reported that none of these agents was used for disinfection or sterilization.

In conclusion, here we report a unique case of diffuse corneal edema secondary to uneventful pterygium surgery. We attribute this steroid-responsive complication to two possible conditions: mild TASS or PVP-I-induced corneal toxicity. During pterygium surgery, caution must be taken to clear all PVP-I. Further studies investigating the penetration of different concentrations of PVP-I and determining the concentration that causes corneal endothelial toxicity or toxic keratopathy should also be performed to understand its pharmacokinetics in damaged cornea.

Informed Consent: Obtained.

Peer-review: Externally peer reviewed.
Authorship Contributions

Surgical and Medical Practices: C.A., B.M., O.K., A.Ö., Concept: C.A., B.M., O.K., A.Ö., B.T.A., A.A., Design: C.A., B.M., O.K., A.Ö., B.T.A., A.A., Data Collection or Processing: C.A., B.M., O.K., A.Ö., B.T.A., A.A., Analysis or Interpretation: C.A., B.M., O.K., A.Ö., B.T.A., A.A., Literature Search: C.A., B.M., O.K., A.Ö., B.T.A., A.A., Writing: C.A., B.M., O.K., A.Ö., B.T.A.

Conflict of Interest: No conflict of interest was declared by the authors.

Financial Disclosure: The authors declared that this study received no financial support.

\section{References}

1. Singh SK. Pterygium: epidemiology prevention and treatment. Community eye Heal. 2017;30:S5-S6.

2. Hacioğlu D, Erdöl H. Developments and current approaches in the treatment of pterygium. Int Ophthalmol. 2017;37:1073-1081.

3. Safianik B, Ben-Zion I, Garzozi HJ. Serious corneoscleral complications after pterygium excision with mitomycin C. Br J Ophthalmol. 2002;86:357-358.

4. Cutler Peck CM, Brubaker J, Clouser S, Danford C, Edelhauser HE, Mamalis N. Toxic anterior segment syndrome: Common causes. J Cataract Refract Surg. 2010;36:1073-1080

5. Otani K, Shimada H, Nakashizuka H, Okubo H. Capsular bag irrigation using $0.025 \%$ povidone-iodine in balanced salt solution PLUS for the treatment of postoperative endophthalmitis. Int Ophthalmol. 2018;38:17871790.

6. Alp BN, Elibol O, Sargon MF, Aslan OS, Yanyali A, Karabas L, Talu H, Caglar Y. The effect of povidone iodine on the corneal endothelium. Cornea. 2000;19:546-550.

7. Naor J, Savion N, Blumenthal M, Assia EI. Corneal endothelial cytotoxicity of diluted povidone--iodine. J Cataract Refract Surg. 2001;27:941-947.

8. Jiang J, Wu M, Shen $\mathrm{T}$. The toxic effect of different concentrations of povidone iodine on the rabbit's cornea. Cutan Ocul Toxicol. 2009;28:119-124.

9. Hayes S, O’Brart DP, Lamdin LS, Doutch J, Samaras K, Marshall J, Meek $\mathrm{KM}$. Effect of complete epithelial debridement before riboflavin-ultraviolet- $A$ corneal collagen crosslinking therapy. J Cataract Refract Surg. 2008;34:657661.

10. Ünal M, Yücel I, Akar Y, Öner A, Altin M. Outbreak of toxic anterior segment syndrome associated with glutaraldehyde after cataract surgery. J Cataract Refract Surg. 2006;32:1696-1701. 